\title{
Oil wastes management: medium optimization for the production of alpha-linolenic acid in Mucor circinelloides
}

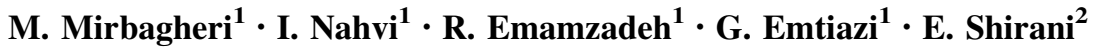

Received: 1 December 2014/Revised: 5 April 2015/Accepted: 5 July 2015/Published online: 5 August 2015

(C) Islamic Azad University (IAU) 2015

\begin{abstract}
The neutraceutical and pharmaceutical application of essential fatty acids is much cleared. Alpha-linolenic acid (ALA) is omega-3 fatty acid and generally known to have beneficial effects in CVS, CNS and other diseases. The purpose of the present investigation is to produce essential fatty acid, especially ALA by Mucor circinelloides from oil wastes. Five oil wastes collected from food industries were used as carbon sources, and the contents of total lipids, biomass and fatty acids were examined during $168 \mathrm{~h}$. The ability of oil waste degradation was determined by measuring of biochemical oxygen demand (BOD) and chemical oxygen demand (COD). Interestingly, $76 \%$ reduction in BOD and $68 \%$ reduction in COD by this strain were achieved, and $M$. circinelloides could be a good candidate for oil waste treatment. In order to enhance ALA production, fermentation variables were chosen in accordance with the fractional design and further optimized by the response surface method. The statistical model was constructed via central composite design. Following the optimization step, ALA production increased by approximately $44.3 \%$, when compared to the screening step. The results indicate that carrying out the fermentation under the conditions of oil waste $4.37 \%$, yeast extract at $0.65 \mathrm{~g} / \mathrm{l}, \quad\left(\mathrm{NH}_{4}\right)_{2} \mathrm{SO}_{4}$ at $0.38 \mathrm{~g} / \mathrm{l}$, an agitation rate of $180 \mathrm{rpm}$ and fermentation time of 3 days will increase the ALA production up to $108.57 \mathrm{mg} / \mathrm{l}$. In this study, a new
\end{abstract}

M. Mirbagheri

maryam_mirbagheri@yahoo.com

1 Department of Biology, Faculty of Science, University of Isfahan, Isfahan 81746-73696, Iran

2 Department of Microbial Biotechnology, Faculty of New Science and Technology, University of Isfahan, Isfahan, Iran renewable source of ALA was employed and optimized successfully for the production of valuable fatty acids.

Keywords Alpha-linolenic acid (ALA) · Biochemical oxygen demand (BOD) · Chemical oxygen demand (COD) . Essential fatty acids $\cdot$ Mucor circinelloides

\section{Introduction}

Essential fatty acids (omega-3/6) have crucial roles in the structure and biological functions required for conserving the homeostasis in all active organisms, which in turn convenes fluidity and modulates the behavior of certain membrane-bound proteins (Gill and Valivety 1997; Vigh et al. 2005). Human health and development has been related to dietary intake of essential fatty acids (Simpoulos 2002). These are precursors for various metabolites such as prostaglandins and leukotrienes and regulating critical biological functions (Gill and Valivety 1997; Dyaneshwar et al. 2006; Yongmanitchai and Ward 1989). The essential fatty acids are important for brain development, immune system function and blood pressure regulation (Avijit et al. 1999; Nakahara et al. 1996; Ratledge 1992; Certik and Shimizu 1999).

Some nuts, seeds and vegetable oils contain alpha-linolenic acid and may be converted to docosahexaenoic acid (DHA) and eicosapentaenoic acid (EPA) in the body (Chapman et al. 1983; Burdge and Calder 2005). Microorganisms are good candidates for essential fatty acid production. Among these, special attention has been paid to the use of various oleaginous zygomycetes fungi like species of Mortierella, Mucor and Cunninghamella and marine fungi like Trichoderma pseudokoningii, Crvalaria lunata and Aspergillus niveus which are capable of 
producing lipids containing linolenic acid (Certik and Shimizu 1999; Devi et al. 2006). Microorganisms can convert not only a broad range of common substrates such as glucose and cellulose, but also agro-residues and some industrial and organic wastes into lipid (Amaral et al. 2012; Chatzifragkou et al. 2010). The fungus Mucor circinelloides was found to be capable of synthesizing EPA when cultivated in thin stillage and centrifuged thin stillage from corn to ethanol production (Liang et al. 2012). The highest concentration of $\gamma$-linolenic acid was obtained with M. circinelloides in culture containing vegetable oil (TaukTornisielo et al. 2009). The contents of total lipids, biomass and various essential fatty acids were examined during $168 \mathrm{~h}$. The ability of oil waste degradation was determined by measuring biochemical oxygen demand (BOD) and chemical oxygen demand (COD) that is novel in this research. We aimed to design an optimal medium for an efficient alpha-linolenic acid (ALA) production using response surface methodology (RSM). RSM is a collection of statistical techniques suitable for experimental design, model construction, evaluating the effects of factors and screening optimum conditions of factors for fascinating responses. Central composite design (CCD) is the most extensively used response surface designs. Although ratability is a desirable property of a central composite design, a face-centered design can be used when there is a difficulty in remaining the start points beyond the experimental region defined by the upper and lower limits of each factor (Box et al. 1978; Haaland 1990). The objective of the present study was using oil wastes as carbon sources for oleaginous zygomycetes fungi $M$. circinelloides DSM1175 for bioconversion of cheap substrate to valuable products like essential fatty acids and pollution control. Various studies have been managed to optimize the production of fatty acids by fungi (Aminah et al. 2006; Mamatha et al. 2008). In the first step, the factorial design was used to investigate the effects of medium components; then, the concentration of these factors was optimized using RSM. This study was performed in a 15-month period between September 2013 and February 2015, on laboratory scale at University of Isfahan.

\section{Materials and methods}

\section{Microorganisms and media}

Mucor circinelloides DSM 1175 (Leibniz Institute DSMZ, Germany) was used for essential fatty acid production. This strain was maintained on potato dextrose agar (PDA) (Merck, Darmstadt, Germany). The inoculated production medium contained (per liter of distilled water) $7.0 \mathrm{~g}$ $\mathrm{KH}_{2} \mathrm{PO}_{4}, 2.5 \mathrm{~g} \mathrm{Na}_{2} \mathrm{HPO}_{4}, 1.5 \mathrm{~g} \mathrm{MgSO}_{4}, 0.06 \mathrm{~g} \mathrm{MnSO}_{4}$,
$0.15 \mathrm{~g} \mathrm{CaCl}_{2}, 0.15 \mathrm{~g} \mathrm{FeCl}_{3}, 0.5 \mathrm{~g}$ yeast extract, $0.5 \mathrm{~g}$ $(\mathrm{NH} 4)_{2} \mathrm{SO}_{4}, \mathrm{pH} 6.0$ and $2 \%(\mathrm{~V} / \mathrm{V})$ oil waste as a carbon source (Papanikolaou et al. 2007). One milliliter of spore suspension (around $1 \times 10^{7}$ spores) was inoculated in 250-ml Erlenmeyer flask containing $50 \mathrm{ml}$ of basal fermentation medium. This was incubated in a rotary shaker incubator at $180 \mathrm{rpm}$ and $28{ }^{\circ} \mathrm{C}$ for $72 \mathrm{~h}$.

\section{Cell dry mass}

The mycelia were harvested from the medium by the filtration through Whatman No. 1 filter paper and thoroughly washed with distilled water. To remove unconsumed oil, an additional washing was carried out using ethanol and then dried at $105{ }^{\circ} \mathrm{C}$ to constant weight. The dry cell weight was measured gravimetrically (Papanikolaou et al. 2004).

\section{BOD and COD determinations}

BOD was determined using the modified iodometric method, and COD was determined by the standard closed reflux method according to the procedures described by Clesceri et al. (1998). The BOD and COD degradation efficiency was defined as the reducing amount of BOD and COD versus the amount of initial values.

\section{Analytical methods}

\section{Extraction and modification of lipids}

Lipid extraction was performed according to the modified procedure of Bligh and Dyer. To assay the lipid and fatty acid content, cells were disrupted by acid hydrolysis and lipid extraction was performed using chloroform/methanol mixture (1:1). The lipid-containing fractions were separated and modified to obtain fatty acid methyl esters (FAMEs) (Pan et al. 2009).

The extracted fatty acids were modified to fatty acid methyl esters (FAMEs) according to the method of Christie (1993). The FAMEs were subjected to analysis using gas chromatography (GC), and the chromatograms were studied based on the standard peaks obtained from the standard FAMEs, which included C18:2 (linolenic acid), C18:3 (GLA), C18:3 (alpha-linolenic acid), C20:4 (ARA), C20:5 (EPA), C22:6 (DHA).

\section{FAMEs analysis by gas chromatography (GC)}

GC was performed on Agilent 19091J-413 series gas chromatograph equipped with a FID and the capillary column HP5 (30 m, 0.25 mm i.d., 0.25- $\mu \mathrm{m}$ film thickness; USA). Injector and detector temperatures were maintained at 260 and $300{ }^{\circ} \mathrm{C}$, respectively. The oven was 
programmed for $2 \mathrm{~min}$ at $100{ }^{\circ} \mathrm{C}$, then increased to $160{ }^{\circ} \mathrm{C}$ at $3 \mathrm{~min}$, maintained for $2 \mathrm{~min}$ at $215^{\circ} \mathrm{C}$, increased further to $217^{\circ} \mathrm{C}$ at $2 \mathrm{~min}$, then maintained for $2 \mathrm{~min}$ at $218{ }^{\circ} \mathrm{C}$ and finally increased to $260{ }^{\circ} \mathrm{C}$ at $2 \mathrm{~min}$. The carrier gas, nitrogen, was used at a flow rate of $1.5 \mathrm{ml} / \mathrm{min}$. The injection volume was $1 \mu \mathrm{l}$, with a split ratio of 100:1.

\section{Experimental design and statistical analysis}

The samples of different oil wastes from various industries including restaurants and fried food factories were collected in Isfahan, Iran. The samples were abbreviated as F1 and F2 for oil wastes from fried food factory of Shilan Kish, F3 for the sample from Naz plant oil factory, and R1 and R2 from two different fast food restaurants. In order to select the significant variables for ALA production, the independent variables such as oil waste, mineral nitrogen sources (ammonium sulfate), organic nitrogen sources (yeast extract), $\mathrm{pH}$ and inoculation were considered and screened via $1 / 2$ fractional factorial design. A total of five variables were included for screening, and each independent variable was tested at two levels: high level $(+1)$ and low level $(-1)$. For a 1/2 fractional factorial with the five variables, 48 experimental runs with three replications of the center points are required. After fractional design, the fundamental factors influencing the essential fatty acid and lipid production were selected and subjected to RSM to obtain maximum concentration and level of individual factors.

Three independent variables, carbon sources (oil wastes) $(A)$, yeast extract $(B)$ and ammonium sulfate concentrations $(C)$, and the dependent response variable ALA, total lipid, biomass, GLA and linoleate were studied. The experimental design of CCD is listed in Table 1. By using this design, the experimental data were fitted according to the equation. The response data obtained after central composite design were analyzed by Minitab version 16 and Design Expert version 7 software which generated 3D contour plots and standard analysis of variance (ANOVA) indicating the optimum concentrations and interaction among these factors.

\section{Results and discussion}

In the present study, the impact of $M$. circinelloides on the production of essential oil, especially ALA, and alterations in BOD and COD was studied. Five oil wastes were used as carbon sources, and biochemical aspects of media such as the total amount of lipids, biomass and essential fatty acids were examined by optimization of media by fractional design and response surface methods.
Table 1 Design of experiments: central composite design of variable

\begin{tabular}{|c|c|c|c|}
\hline Run number & Oil waste $(\%)$ & Yeast extract $(\mathrm{g} / \mathrm{l})$ & $\left(\mathrm{NH}_{4}\right)_{2} \mathrm{SO}_{4}(\mathrm{~g} / \mathrm{l})$ \\
\hline 1 & 4.00 & 0.55 & 0.55 \\
\hline 2 & 4.00 & 1.31 & 0.55 \\
\hline 3 & 9.05 & 0.55 & 0.55 \\
\hline 4 & 4.00 & 0.55 & 0.55 \\
\hline 5 & 7.00 & 1.00 & 1.00 \\
\hline 6 & 4.00 & 0.55 & 0.55 \\
\hline 7 & 4.00 & 0.55 & 0.55 \\
\hline 8 & 1.00 & 0.10 & 0.10 \\
\hline 9 & 4.00 & 0.55 & 1.31 \\
\hline 10 & 4.00 & 0.55 & -0.21 \\
\hline 11 & 7.00 & 0.10 & 0.10 \\
\hline 12 & 1.00 & 1.00 & 0.10 \\
\hline 13 & 1.00 & 1.00 & 1.00 \\
\hline 14 & 1.00 & 0.10 & 1.00 \\
\hline 15 & 4.00 & 0.55 & 0.55 \\
\hline 16 & 4.00 & -0.21 & 0.55 \\
\hline 17 & 7.00 & 1.00 & 0.10 \\
\hline 18 & -1.05 & 0.55 & 0.55 \\
\hline 19 & 7.00 & 0.10 & 1.00 \\
\hline
\end{tabular}

\section{Lipid and fatty acid determination}

To assay the lipid and fatty acid content, cells were disrupted and lipid extraction was performed subsequently. The FAMEs were subjected to analysis using gas chromatography (GC). Table 2 shows a comparison between the produced lipids, biomass, yield of production (rate of lipid to biomass) and essential fatty acids by the strain in medium containing different oil wastes. The results show that R1 is the main substrate for lipid production and its final product is about $60 \%$. Furthermore, ALA is the important fatty acid that produces by the strain. The onset of lipid turnover occurred rapidly after transition from nitrogen-limitation to carbon-starvation conditions (Fakas et al. 2008; Kendrick and Ratledge 1992). Zygomycete fungi like Mortierella spp., Rhizopus spp. and Cunninghamella sp. are good strains for essential fatty acids production, but ALA was found in low concentrations in the produced oils (Certik and Shimizu 1999; Chatzifragkou et al. 2010; Jangbua et al. 2009). However, in M. circinelloides DSM 1175, when large amounts of oil were accumulated in the mycelium, ALA was present in large concentrations (Table 2).

\section{BOD and COD assay}

BOD and COD removal by the strain in oil wastes was significant when compared with control. Comparison of 
Table 2 Comparison of lipid(g/g), biomass(g/l), yields (\%lipid/biomass w/w), essential fatty acid production (mg/g) by two Mucor circinelloides in media containing different oil wastes as carbon sources (F1, 2, 3 oil wastes from factories and R1, 2 oil wastes from restaurant)

\begin{tabular}{|c|c|c|c|c|c|c|c|}
\hline \multirow{2}{*}{$\begin{array}{l}\text { Oil waste in } \\
\text { media }\end{array}$} & \multirow{2}{*}{$\begin{array}{l}\text { Biomass } \\
(\mathrm{g} / \mathrm{l})\end{array}$} & \multirow{2}{*}{$\begin{array}{l}\text { Total lipid } \\
(\mathrm{g} / \mathrm{g})\end{array}$} & \multirow{2}{*}{$\begin{array}{l}\text { Yield (w/ } \\
\text { w \%) }\end{array}$} & \multicolumn{4}{|c|}{ Essential fatty acid concentrations in total lipid $(\mathrm{mg} / \mathrm{g})$} \\
\hline & & & & $\begin{array}{l}\text { C18:2 } \\
\text { linoleate (n-6) }\end{array}$ & $\begin{array}{l}\text { C18:3 gamma linoleate } \\
\text { (GLA) (n-6) }\end{array}$ & $\begin{array}{l}\text { C } 18: 3 \\
\text { linoleate }(n-3)\end{array}$ & $\begin{array}{l}\text { C22:6 docosahexaenoate } \\
\text { (DHA) (n-3) }\end{array}$ \\
\hline F1 & 10.3 & 4.2 & 40.7 & 84.22 & 10.85 & 78.6 & 6.78 \\
\hline $\mathrm{F} 2$ & 13.78 & 5.1 & 37 & 15.15 & 7.35 & 64.5 & - \\
\hline F3 & 12.49 & 4.3 & 34.42 & 64.8 & 3.131 & 34.3 & - \\
\hline $\mathrm{R} 1$ & 10.63 & 6.4 & 60.21 & 75.85 & 13.4 & 91.4 & 3.23 \\
\hline $\mathrm{R} 2$ & 11.73 & 4.92 & 35 & 50.12 & 12.48 & 87.5 & 7.21 \\
\hline
\end{tabular}

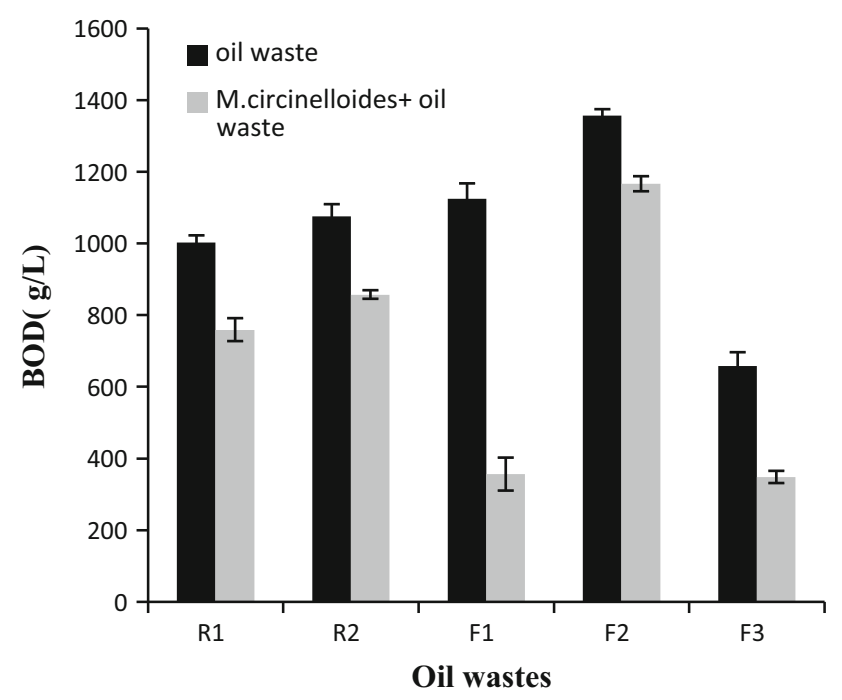

Fig. 1 Biochemical oxygen demand $\left(\mathrm{BOD}_{5}\right)$ values $(\mathrm{g} / \mathrm{l})$ in different oil wastes and oil wastes treatment by oleaginous fungus $M$. circinelloides DSM 1175 (F1, 2, 3 oil wastes from factories and R1.2 oil wastes from restaurant)

$\mathrm{BOD}_{5}$ and $\mathrm{COD}$ in oil wastes and oil waste treated by M. circinelloides is shown in Figs. 1,2. Maximum reduction percent of BOD and COD was about 68 and $76 \%$ in different oil waste, respectively. Tomato waste hydrolyses was used by these fungi for fatty acid production (Fakas et al. 2008). Food waste, olive oil mill wastewaters, industrial fats and whey and starch hydrolyses were other wastes for microbial lipid production by oleaginous fungi (Amaral et al. 2012; Fakas et al. 2008; Zhu et al. 2003). Strong (2009) used four fungi to reduce COD in distillery wastewater. This study determined that using fungi could reduce oil waste pollutions and production of valuable products.

\section{Optimization by using the factorial design}

The results of the $1 / 2$ fractional factorial design indicate that three factors including yeast extract, $\left(\mathrm{NH}_{4}\right)_{2} \mathrm{SO}_{4}$ and

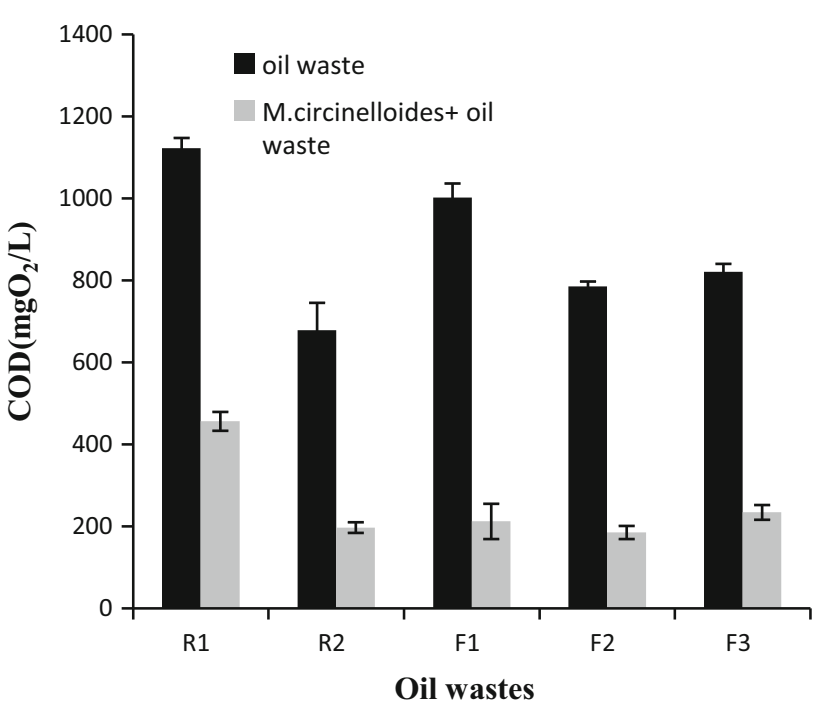

Fig. 2 Chemical oxygen demand (COD) values $\left(\mathrm{mgO}_{2} / \mathrm{l}\right)$ in different oil wastes and oil wastes treatment by oleaginous fungus $M$. circinelloides DSM 1175 (F1, 2, 3 oil wastes from factories and R1.2 oil wastes from restaurant)

carbon sources have a significant effect on ALA production by $M$. circinelloides. Figure 3 indicates that three factors including $\mathrm{pH}$ and the inoculation have not significant effects on ALA production by the strain. The analysis of variance of the variables on the response in the screening step confirmed these results (not showed). The $p$ values less than 0.05 were considered to show a significant effect on the response.

\section{Optimization of the significant variables using RSM}

Results showed that carbon source, yeast extract and ammonium sulfate were the major nutrients for the enhancement of ALA. The data obtained were subjected to regression analysis using the second-order regression equation. The coefficients of the regression equation were calculated using the Design Expert software provided in the following regression equation. 


\section{Normal Plot of the Effects}

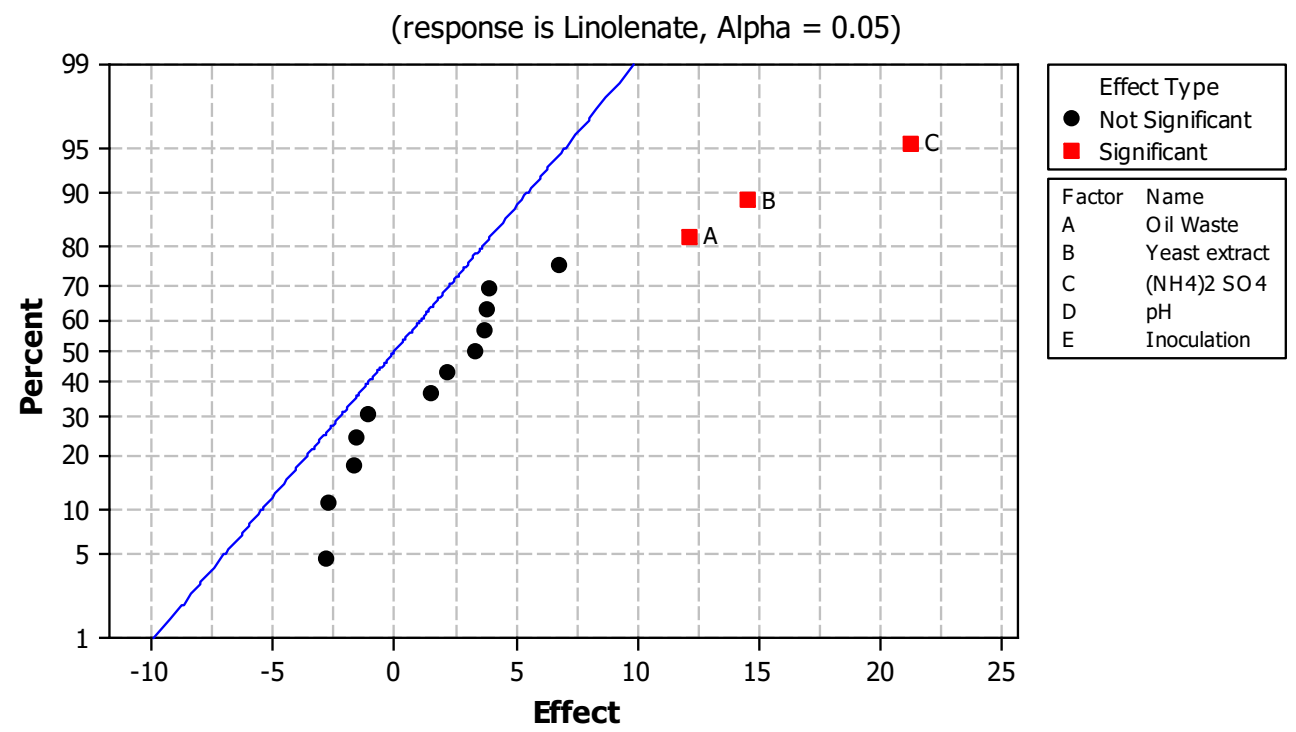

Lenth's PSE $=4.2375$

Fig. 3 Normal plot of effective factors in alpha-linolenic acid production by $M$. circinelloides DSM 1175

$$
\begin{aligned}
Y= & 98.42+18.61 A+16.22 B-15.74 C+16.39 A B \\
& +14.7 A C-1.28 B C-A^{2}-19.66 B^{2}-6.99 C^{2}
\end{aligned}
$$

where $Y$ is ALA concentration, $A$ oil waste concentration, $B$ yeast extract concentration and $C\left(\mathrm{NH}_{4}\right)_{2} \mathrm{SO}_{4}$. The results of the second-order regression model fitting in the form of ANOVA are given in Table 3. A good fit of the regression model was checked by the coefficient of determination $\left(R^{2}\right)$, correlation coefficient $(R)$ values and total regression $F$ test. This is an estimate of the fraction of the overall variation in the data accounted by the model, and thus, the model is capable of explaining $89.1 \%$ of the variation in response. It ensures a satisfactory adjustment of the quadratic model to the experimental data. The 'adjusted $R^{2}$, was found to be 0.7821 , indicating that the model is good. Also, the 'model $F$ value' of 49.6 implies that the model is significant. The ANOVA results (Table 3) indicated that the independent variable oil waste is influencing the ALA production very significantly $(p<0.05)$.

The contour plots and 3D response surface curves were created by plotting the response against each of the two independent variables for determination of optimal levels of each variable for maximum ALA production, while maintaining the other variables at their fixed (zero) levels. The main interaction effects of these two factors were understood by this manner. These plots were easily
Table 3 ANOVA for RSM analysis for response linoleate (ALA) surface quadratic model obtained from experimental designs

\begin{tabular}{lrrrrrl}
\hline Source & Sum of squares & $d f$ & Mean square & $F$ value & $p$ value prob. $>F$ & Significance \\
\hline Model & $23,840.83$ & 9 & 2648.98 & 8.19 & 0.0022 & Significant \\
A-oil waste & 4728.71 & 1 & 4728.71 & 14.62 & 0.0041 & Significant \\
B-yeast extract & 3593.94 & 1 & 3593.94 & 11.11 & 0.0088 & Significant \\
C- $\left(\mathrm{NH}_{4}\right) 2 \mathrm{SO}_{4}$ & 3384.75 & 1 & 3384.75 & 10.46 & 0.0102 & Significant \\
$\mathrm{AB}$ & 2149.91 & 1 & 2149.91 & 6.65 & 0.0298 & Significant \\
$\mathrm{AC}$ & 1727.72 & 1 & 1727.72 & 5.34 & 0.0462 & Significant \\
$\mathrm{BC}$ & 13.12 & 1 & 13.12 & 0.041 & 0.8449 & Not significant \\
$A^{2}$ & 4082.82 & 1 & 4082.82 & 12.62 & 0.0062 & Significant \\
$B^{2}$ & 5274.05 & 1 & 5274.05 & 16.30 & 0.0029 & Significant \\
$C^{2}$ & 667.68 & 1 & 667.68 & 2.06 & 0.1847 & Not significant \\
Residual & 2911.79 & 9 & 323.53 & & & \\
Pure error & 46.22 & 4 & 11.55 & & & \\
Cor total & $26,752.62$ & 18 & & & & \\
\hline
\end{tabular}


obtained by calculating from the model values taken by one factor where the second factor varies with restriction of a given $Y$ value.

The yield values for different concentrations of the variables are also predicted from the respective response surface plots (Figs. 4, 5, 6). The maximum predicted yield was indicated by the surface confined in the response surface diagram.

The response surface plot was obtained as a function of oil waste concentration versus yeast extract concentration, while the third variable ammonium sulfate was maintained at zero level (coded; Fig. 4). An increase in ALA yield with an increase in concentration of oil waste versus yeast extract was observed. Among the two independent variables, oil waste plays a major role in the production of ALA. Its level was very critical as evidenced from the 3D surface graph. The optimum value was near the center point of oil waste level, and the interaction of oil waste and ammonium sulfate did not result in any further beneficial effect on the system. The response surface plot was obtained as a function of concentration of oil waste versus yeast extract when the third variable ammonium sulfate was kept at its mid-level (Fig. 5). An increased ALA with increased concentration of oil waste versus ammonium sulfate was also observed. As indicated in Fig. 1a, the same trend was also observed here. The role of oil waste is very critical and significant when compared with ammonium sulfate. The interaction effect of both independent variables resulted in a decrease in the production of ALA after the mid-level. The effect of concentration of yeast extract versus ammonium sulfate, when the third variable is oil waste, was maintained at zero level and not significant (Fig. 6). An increase in ALA yield with increased concentration of ammonium sulfate was also observed. From the response surface, ANOVA revealed that the increase in concentration of ammonium sulfate or yeast extract was

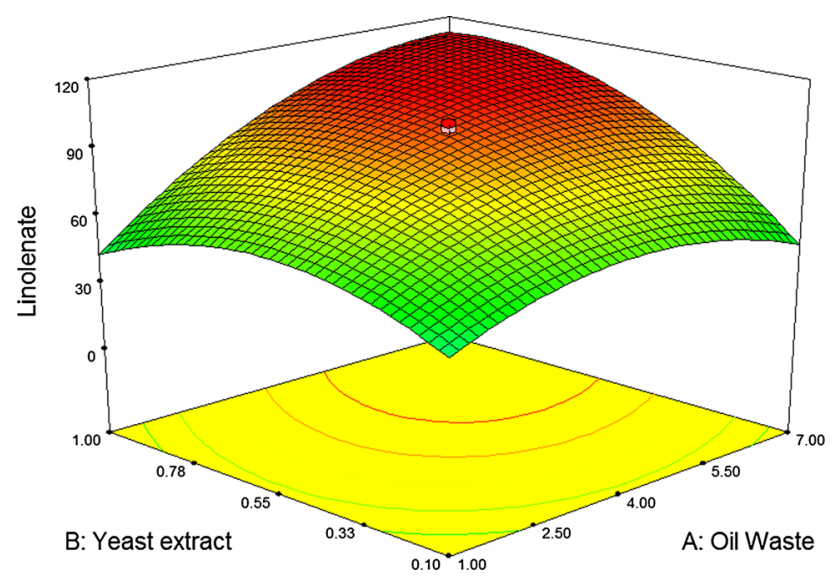

Fig. 4 Contour plot of ALA productivity (g/l) as a function of oil waste $(\mathrm{g} / \mathrm{l})$ and yeast extract $(\mathrm{g} / \mathrm{l})$ concentrations

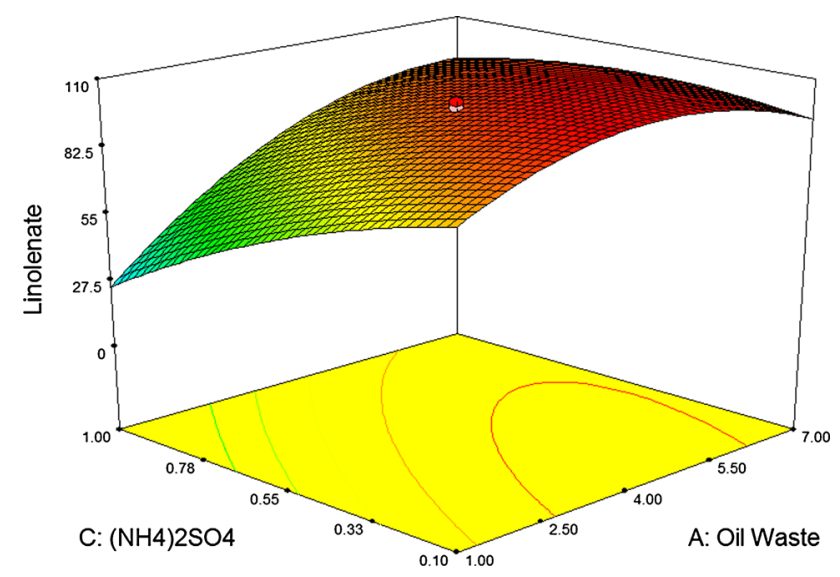

Fig. 5 Contour plot of ALA productivity ( $g / l)$ as a function of oil waste $(\mathrm{g} / \mathrm{l})$ and ammonium sulfate $(\mathrm{g} / \mathrm{l})$ concentrations

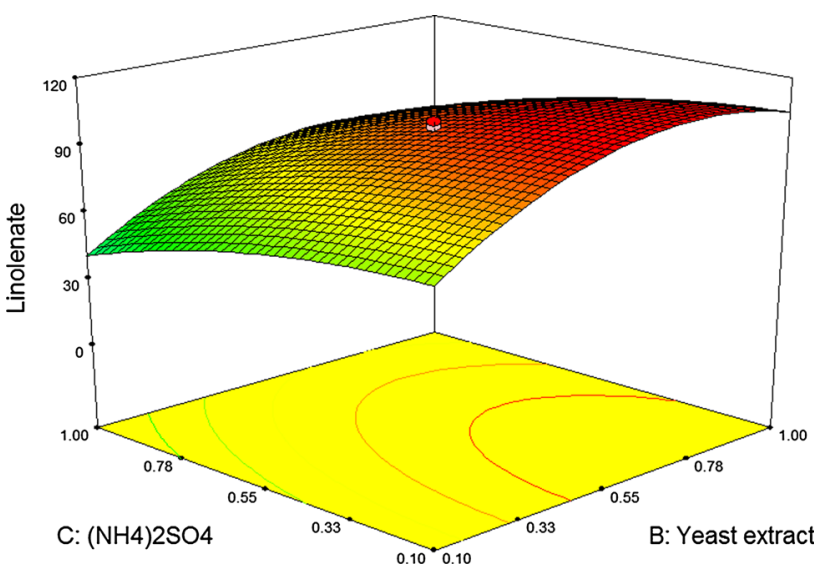

Fig. 6 Contour plot of ALA productivity ( $g / 1)$ as a function of yeast extract $(\mathrm{g} / \mathrm{l})$ and ammonium sulfate $(\mathrm{g} / \mathrm{l})$ concentrations

only marginal for ALA increase in the total lipid of mycelium.

The results supported the predicted values and the effectiveness of the model. A $10.4 \%$ increase in production of ALA was achieved when compared to that in the basal medium. The results of the conditions for maximizing the ALA production within the experimental region were predicted and given. The results were validated by doing the experiments with the predicted levels by the model. The results indicated that the predicted and observed values for ALA did not differ significantly $(p>0.05)$. This indicated that the optimized medium components concentration favored the production of ALA in the mycelium. Figure 7 represents the differences between actual and predicted responses that completed results in Table 3. These results cleared that the model is acceptable for biomass, GLA and linoleate like ALA which are explained in this paper. In this study, low production of ALA was obtained by low concentration of nutrients. In contrast, inhibition of secondary metabolite synthesis resulted in 


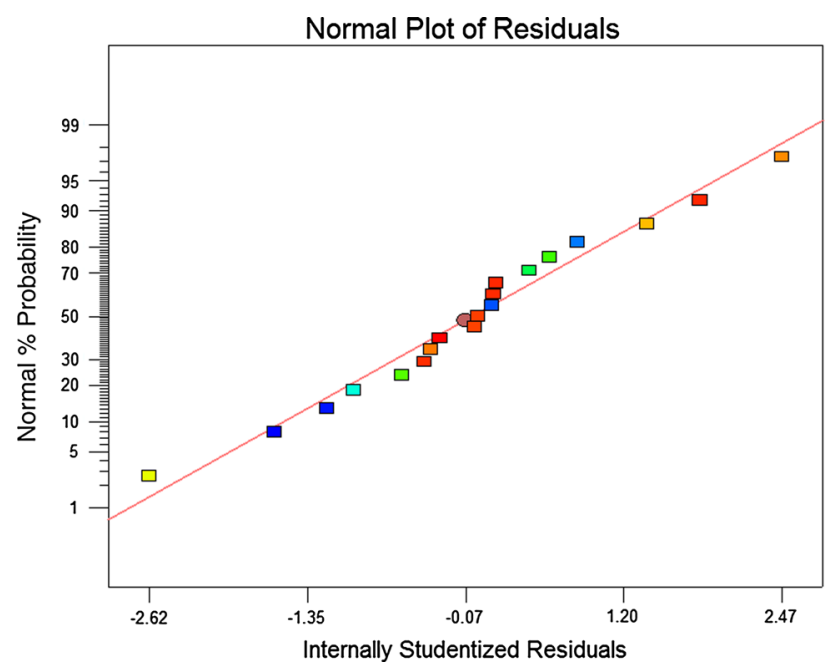

Fig. 7 Representation of predicted versus actual response of experimental runs under RSM design

higher concentration (ALA increase in the fatty acids). This was observed in other findings on carbon and nitrogen repression effects (Certik and Shimizu 1999; Ratledge 1992). To obtain optimum yield of secondary metabolite production in this work, a multifactorial statistical method was used for showing the conditions. CCD exploits the amount of information that can be obtained while seeing the interaction of independent variables and limiting the number of individual experiments required. The response surface methodology that is commonly satisfying the optimization of many microbial processes is a smaller and less time-consuming experimental design (Chang et al. 2006; Linder et al. 2005). Aminah et al. (2006), Mamatha et al. (2008), and Rocky-Salimi et al. (2011) applied RSM for optimization of fatty acid production by fungi. The ideal culture medium found in this work could be applied as a basis for further study with batch or fed-batch cultivation.

\section{Conclusion}

For determination of the conditions causing the maximum yield of ALA, the RSM was found to be functional. The use of an experimental design allowed the rapid screening of a large experimental domain in search of the best condition and levels of the ALA production. To the best of our knowledge, there were no reports available on the ALA production by media engineering. This research focused chiefly on an attempt to reveal the application of statistical design and RSM to maximize ALA production. Using cheap substrate like oil wastes and reduction in BOD and COD were the other findings of this work.
Acknowledgments The authors would like to thank Dr Parvaneh Rahimi-Moghaddam M.D., Ph.D., Department of Pharmacology, College of Medicine, Iran University of Medical Sciences, and Gholamreza Ghezelbash and Hossein Ghanavati Ph. D students in University of Isfahan for their guidance and University of Isfahan for financially supporting this project.

\section{References}

Amaral C, Marcos L, Sampaio A, Peres J, Dias AA, Peixoto F, Anjos M, Pais C (2012) Biodegradation of olive mill wastewaters by a wild isolate of Candida oleophila. Int Biodeterior Biodegrad 68:45-50

Aminah S, Aidil SA, Wan Mohtar WY (2006) Medium optimization for the production of lipidless biomass by Cunninghamella sp. 2A1 using response surface methodology. Malays J Microbiol 2:40-45

Avijit H, Tripathi SK, Ghosh A (1999) Pharmacology and therapeutic potential of the $n-3$ polyunsaturated fatty acids, eicosapentaenoic acid (EPA) and docosahexaenoic acid (DHA) in fish oils. Indian J Pharmacol 31:247-264

Box GEP, Hunter SW, Hunter JS (1978) Statistic for experiments an introduction to design, data analysis and model building. Wiley, New York

Burdge GC, Calder PC (2005) Conversion of alpha-linolenic acid to longer-chain polyunsaturated fatty acids in human adults. Reprod Nutr Dev 45:581-597

Certik M, Shimizu S (1999) Biosynthesis and regulation of microbial polyunsaturated fatty acid production. J Biosci Biotechnol 87:1-14

Chang CY, Lue MY, Pan TM (2006) Statistical optimization of medium components for the production of Antrodia cinnamomea AC0623. Appl Microbiol Biotechnol 72:654-661

Chapman DJ, De-Felice J, Barber J (1983) Growth temperature effects on thylakoid membrane lipid and protein content of pea chloroplasts 1. Plant Physiol 72:225-228

Chatzifragkou A, Fakas S, Galiotou-anayotou M, Komaitis M, Aggelis G, Papanikolaou S (2010) Commercial sugars as substrates for lipid accumulation in Cunninghamella echinulata and Mortierella isabellina fungi. Eur J Lipid Sci Technol 112:1048-1057

Christie WW (1993) Preparation of ester derivatives of fatty acids for chromatographic analysis. In: Christie WW(ed) Gas chromatography and lipids. Oily Press, Dundee, pp 69-111

Clesceri LS, Greenberg AE, Eaton AD (1998) Standard methods for the examination of water and wastewater. American Public Health Association (APHA), Washington, DC

Devi P, Divy A, Shridhar MP, Dsouza L, Naik CG (2006) Cellular fatty acid composition of marine derived fungi. Indian J Mar Sci 35:359-363

Dyaneshwar W, Kalpana J, Abhay H (2006) Polyunsaturated fatty acids: biotechnology. Crit Rev Biotechnol 26:83-93

Fakas S, Certik M, Papanikolaou S, Aggelis G, Komaitis M, Galiotou- Panayotou M (2008) $\gamma$-Linolenic acid production by Cunninghamella echinulata growing on complex organic nitrogen sources. Bioresour Technol 99:5986-5990

Gill I, Valivety R (1997) Polyunsaturated fatty acids, part 1: occurrence, biological activities and applications. Trends Biotechnol 15:401-409

Haaland PD (1990) Experimental design in biotechnology. Elsevier Science, New York

Jangbua P, Laoteng K, Kitsubun P, Nopharatana M, Tongta A (2009) Gamma-linolenic acid production of Mucor rouxii by solid-state fermentation using agricultural by-products. Lett Appl Microbiol 49:91-97 
Kendrick A, Ratledge C (1992) Lipids of selected moulds grown for production of n-3 and n-6 polyunsaturated acids. Lipids 27:15-20

Liang Y, Wen Z, Strait M (2012) Use of dry milling derived thin stillage for producing EPA by the fungus Pythium irregulare. Bioresour Technol 111:404-409

Linder M, Kochanowski N, Fanni J, Armentier M (2005) Response surface optimization of lipase catalyzed esterification of glycerol and n-3 polyunsaturated fatty acids from salmon oil. Process Biochem 40:273-279

Mamatha SS, Ravi R, Venkateswaran G (2008) Medium optimization of gamma linolenic acid production in Mucor rouxii CFR-G15 using RSM. Food Bioprocess Technol 1:405-409

Nakahara T, Yokochi Y, Higashihara T, Tanaka S, Yaguchi T, Honda D (1996) Production of docosahexaenoic and docosapentaenoic acid by Schizochytrium sp. isolated from Yap Islands. J Am Oil Chem Soc 73:1421-1426

Pan L, Yang D, Shao L, Li W, Chen G, Liang Z (2009) Isolation of oleaginous yeasts. Food Technol Biotechnol 47:215-220

Papanikolaou S, Sarantou S, Komaitis M, Aggelis G (2004) Repression of reserve lipid turnover in Cunninghamella echinulata and Mortierella isabellina cultivated in multiple-limited media. J Appl Microbiol 97:867-875

Papanikolaou S, Galiotou-Panayotou M, Fakas S, Aggelis G (2007) Lipid production by oleaginous Mucorales cultivated on renewable carbon sources. Eur J Lipid Sci Technol 109:1060-1070
Ratledge C (1992) Microbial lipids: commercial realities or academic curiosities. In: Kyle DJ, Ratledg C (eds) Industrial applications of single cell oils. AOCS, IL, pp 1-15

Rocky-Salimi K, Hamidi-Esfahani H, Abbasi S (2011) Statistical optimization of arachidonic acid production by Mortierella alpine CBS 754.68 in submerged fermentation. Iran J Biotechnol 9:87-95

Simpoulos AP (2002) The importance of the ratio of omega 6/omega 3 essential fatty acids. Biomed Pharmacother 56:365-379

Strong PG (2009) Fungal remediation of Amarula distillery wastewater. World J Microbiol Biotechnol 26:133-144

Tauk-Tornisielo S, Arasato LS, Almeida A, Govone JS, Malagutti EN (2009) lipid formation and g-linolenic acid production by Mucor circinelloides and Rhizopus sp., grown on vegetable oil. Braz J Microbiol 40:342-345

Vigh L, Escriba PV, Sonnleitner A, Sonnleitner M, Piotto S, Maresca B, Horvath I, Harwood JL (2005) The significance of lipid composition for membrane activity: new concepts and ways of assessing function. Prog Lipid Res 44:303-344

Yongmanitchai W, Ward OP (1989) Omega-3 fatty acids: alternative sources of production. Process Biochem 24:117-125

Zhu M, Yu L, Wu Y (2003) An inexpensive medium for production of arachidonic acid by Mortierella alpina. J Ind Microbiol Biotechnol 30:75-79 